\title{
Frühe Bauchlage erhöht Überlebenschance
}

Fragestellung: Beeinflusst eine frühzeitige Bauchlagerung beatmeter Patienten mit akutem progressiven Lungenversagen (ARDS) das Outcome günstig?

Hintergrund: Die Positionierung von Patienten mit ARDS in Bauchlage ist seit Jahren eine Vorgehensweise, die angewendet wird, um physiologische Surrogatmarker, vorrangig die Oxygenierung, zu verbessern. Auch in randomisierten Studien konnte im Vergleich zur normalen Rückenlage durch eine Bauchlagerung eine signifikante Verbesserung der Oxygenierung bestätigt werden. Allerdings ließ sich dieser Aspekt nicht in ein günstigeres Outcome übertragen. Metaanalysen hatten jedoch die Vermutung nahegelegt, dass sich zumindest die Überlebensrate durch eine Bauchlagerung steigern lassen könnte.

Patienten und Methodik: In dieser prospektiven multizentrischen kontrollierten Studie wurden auf 27 Intensivstationen insgesamt 474 Patienten mit schwerem, seit über 36 Stunden bestehenden ARDS in entweder Rücken- oder Bauchlagerung randomisiert. Die Bauchlage wurde frühzeitig begonnen und für mindestens 16 Stunden kontinuierlich aufrechterhalten. Ein ARDS wurde entsprechend der Amerikanisch-Europäischen Konsensuskonferenz-Kriterien defi-

Guérin C., et al. for the PROSEVA

Study Group. Prone Positioning

in Severe Acute Respiratory Distress Syndrome. N Engl J Med

2013; 368: 2159-68 niert mit einem $\mathrm{FiO}_{2}$ von mindestens $60 \%$, einem PEEP von mehr als $5 \mathrm{~mm}$ Wassersäule, einem Tidalvolumen von $6 \mathrm{ml}$ pro Kilogramm Körpergewicht sowie einem $\mathrm{PaO}_{2}$-l
$\mathrm{FiO}_{2}$-Verhältnis von $<150 \mathrm{mmHg}$. Primärer Endpunkt war Mortalität am Tag 28, sekundäre Endpunkte waren die Mortalität nach drei Monaten sowie die Rate erfolgreicher Extubationen, Dauer des Intensivaufenthaltes und Komplikationen.

Ergebnisse: Die Bauchlagerung wurde im Schnitt $4 \pm 4$ Mal pro Patient für rund $17 \pm 3$ Stunden in diesem Behandlungsarm durchgeführt. Die Basisparameter der eingeschlossenen Patienten in beiden Vergleichsgruppen waren ähnlich bis auf einen leicht erhöhten SOFA-Wert (Sepsis, Organversagen) zu ungunsten der Rückenlagegruppe. Die Hauptursache des ARDS war eine Pneumonie (rund 60\%), viele der Patienten benötigten Vasopressoren und Muskelrelaxation, bis zu $45 \%$ erhielten Glukokortikoide. Hinsichtlich des primären Endpunktes zeigte sich eine signifikante Reduktion der 28-Tage-Mortalität in der Patientengruppe mit Bauchlagerung ( $16 \%$ versus $32,8 \%, \mathrm{p}<0,001$ ). Ferner war die Mortalität nach drei Monaten deutlich geringer $(23,6 \%$ versus $41 \%, p<0,001)$. Auch nach Adjustierung des initialen klinischen Schweregrades (SOFA-Wertes) bestätigte sich dieses Ergebnis, das sich statistisch auf eine Number-neededto-treat (NNT) von 6 übertragen lässt. Die Komplikationsraten waren in beiden Armen vergleichbar, die Rate an Herzstillständen sogar etwas geringer in der Bauchlagerungsgruppe.

Schlussfolgerungen: Die PROSEVA-Studie zeigte einen deutlichen Überlebensvorteil schwer betroffener ARDS-Patienten durch eine Bauchlagerung auf. Insbesondere wenn die Bauchlagerung frühzeitig und auch länger anhaltend durchgeführt wird, verbessert dies signifikant die Überlebenswahrscheinlichkeit.

\section{- Kommentar von Hagen B. Huttner, Erlangen}

\section{Diese einfache Maßnahme bei den Intensivteams etablieren}

Vorherige ARDS-Interventionsstudien, die beispielsweise Prostacycline, Beta-2-Mimetika, Surfactant, Statine, Omega-3-Fettsäuren oder Rheologika getestet hatten, berichteten zwar über positive Effekte auf Surrogatparameter, wie die Oxygenierung, jedoch handelte es sich - auch bei früheren Bauchlagerungsstudien - bislang fast nur um Negativstudien hinsichtlich des Outcomes. Die Ergebnisse dieser Arbeit sind in der erzielten Klarheit nicht erwartet worden und erscheinen fast "zu gut um wahr zu sein" - eine Mortalitätssenkung von über $50 \%$ durch eine relativ einfache, nicht pharmakologische Intervention ist absolut bahnbrechend! Es muss natürlich die Frage aufgeworfen werden, warum denn frühere Bauchlagerungsstudien keinen klinischen Nutzen identifizieren konnten? Anscheinend haben die frühzeitige Intervention und die tatsächlich lange durchgeführte Bauchlagerung bei - in dieser Studie - wirklich schwer betroffenen ARDS-Patienten nachhaltig positive Effekte. Allerdings sollte angemerkt werden, dass die behandelnden Intensivteams sehr erfahrene Lungenspezialisten sind und somit die Übertragbarkeit der Ergebnisse auf sämt- liche Intensivstationen schwierig ist. Die Intervention ist prinzipiell einfach und erlernbar, ohne dass teures Equipment angeschafft werden muss, und - basierend auf den berichteten Mortalitätseffekten - unglaublich wirkungsvoll. Es sollten alle Anstrengungen unternommen werden, das Personal von Intensivstationen in diesem Feld weiter zu bilden, um eigene ARDSPatienten bestmöglich zu behandeln.

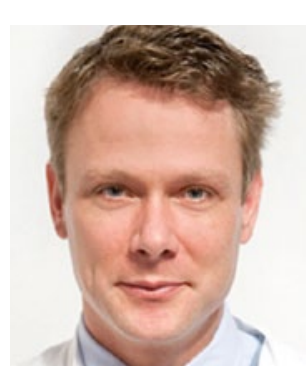

PD Dr. med. Hagen B. Huttner, Erlangen

Universitätsklinik für Neurologie, Erlangen E-Mail: hagen.huttner@uk-erlangen.de 